\title{
PRO 2000: next steps for microbicide development
}
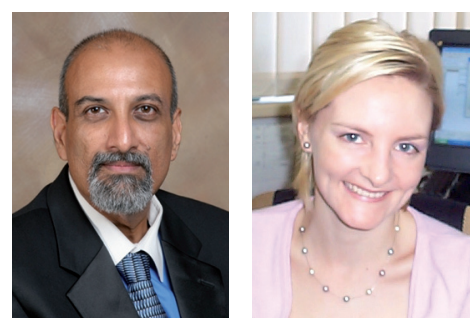

"...the HPTN 035 trial demonstrated that ... 0.5\% PRO 2000 gel resulted in a $30 \%$ reduction in HIV infection in an intent-to-treat analysis..."

Salim S Abdool Karim ${ }^{\dagger} \&$ Cheryl Baxter

${ }^{\dagger}$ Author for correspondence: CAPRISA, University of KwaZulu-Natal, Durban, South Africa = Tel.: +27 312604550 m Fax: +27 312604549 m karims1@ukzn.ac.za

A HIV prevention strategy that women can initiate or control in the form of a microbicide was first proposed almost two decades ago. Since then, various candidate microbides have entered effectiveness trials to assess their impact on the prevention of HIV infection. These include trials of nonoxynol-9 (N9) sponge [1], N9 film [2], N9 gel (COL-1492) [3], SAVVY ${ }^{\circledR}$ (Biosyn, MA, USA) [4], cellulose sulfate (CONRAD, VA, USA) [5], Carraguard ${ }^{\circledR}$ (Population Council, NY, USA) [6] and, most recently, the HIV Prevention Trials Network (HPTN 035) trial that assessed the safety and effectiveness of BufferGel ${ }^{\mathrm{TM}}$ (ReProtect, MD, USA) and $0.5 \%$ PRO 2000 gel (Endo Pharmaceuticals Solutions, DE, USA) for the prevention of male-to-female HIV transmission [7]. Out of all these products only $0.5 \%$ PRO 2000 gel has been able to demonstrate any reduction in HIV infection; three products have shown no impact on HIV (N9 film, Carraguard and BufferGel), one trial did not produce a meaningful result owing to lower than expected HIV incidence in the study population (SAVVY) and three trials suggested that the product may be harmful (N9 sponge, N9 gel and cellulose sulfate).

The results from the HPTN 035 trial demonstrated that, although not statistically significant, $0.5 \%$ PRO 2000 gel resulted in a $30 \%$ reduction in HIV infection in an intent-to-treat analysis and a subgroup analysis provided further corroborating data for a potential protective effect of the gel [7]. These results show promise for the field of microbicides since they are the first indication that a microbicide gel can at least partially reduce a woman's risk of becoming infected with HIV. These results have also reinforced the idea that coitally related products can prevent HIV and have rekindled interest in polymer products. However, these results are insufficient to make any conclusive statement on $0.5 \%$ PRO 2000 gel as a protective microbicide and further trials are needed to provide a clearer indication of the impact of $0.5 \%$ PRO $2000 \mathrm{gel}$ on HIV infection. A larger Phase III effectiveness trial of $0.5 \%$ PRO 2000 gel in the Microbicide Development Program (MDP 301) study of 9389 women is currently underway and its results are expected in late 2009.

Even if the MDP 301 study provides further evidence that $0.5 \%$ PRO 2000 gel can protect against HIV there will still be a strong need for other microbicide candidates and indeed, other HIV prevention options. Successful microbicides will probably be delivered in many forms, such as gels, creams, suppositories, films, sponges and vaginal rings. Some will be contraceptive while others not [8].

\section{Current state of clinical development of microbicides}

There are several candidate microbicides in various stages of development; it is worth noting that testing the safety and efficacy of candidate microbicide products takes many years. According to the Alliance for Microbicide Development, there are currently more than 50 candidate microbicide products in preclinical development and 11 products are being tested in 21 ongoing trials. In addition, a further 16 clinical trials are planned [101].

PRO 2000 gel and tenofovir gel are the candidate microbicides in the most advanced stages of effectiveness testing. PRO 2000 gel is a polyanionic polymer consisting of alternating 2-naphthalene sulfonic acid sodium salt and methylene units, and is an entry/fusion inhibitor designed to hamper HIV's ability to attach to, and infect, healthy cells [102]. In comparison, tenofovir is an antiretroviral-based microbicide, manufactured by Gilead Sciences, Inc. (CA, USA), and licensed to CONRAD. Tenofovir

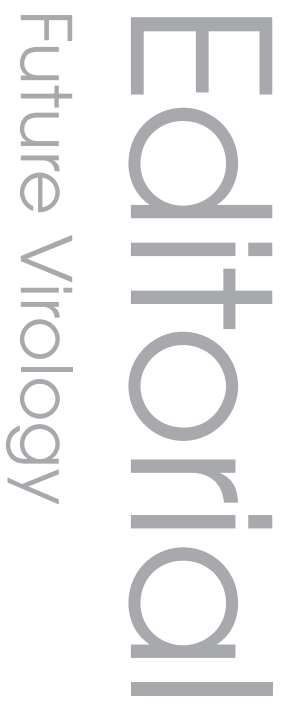

"PRO 2000 gel and
tenofovir gel are
the candidate
microbicides in the most
advanced stages of
effectiveness testing."

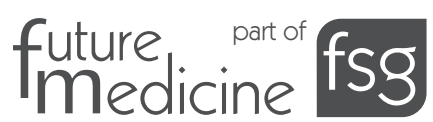




"The pipeline of new
products is growing too
slowly; beyond VivaGel ${ }^{\circledR} .$.
and dapivirine, there are
no products likely to enter
Phase llb and III trials
in the near future."

"The pipeline of new products is growing too slowly; beyond VivaGel ${ }^{\circledR}$... and dapivirine, there are o products likely to enter

in in the near future." has potent activity against retroviruses and interrupts replication of the virus once it enters cells [9]. Tenofovir is already widely used for the treatment of AIDS.

The pipeline of new products is growing too slowly; beyond VivaGel ${ }^{\circledR}$ (Starpharma, Australia) and dapivirine (International Partnership for Microbicides, VA, USA), there are no products likely to enter Phase IIb and III trials in the near future. Early developmental research is also starting to focus on combination microbicides that will include multiple active ingredients or mechanisms of action, but these have extremely long timelines, as each component of the combination may have to demonstrate effectiveness to warrant inclusion in a combination product. Microbicide candidates currently in clinical trials are summarized in TABLE 1.

\section{Obstacles to}

\section{microbicide development}

Several obstacles continue to hamper the development of a safe and effective microbicide. Financial commitments have been one of the biggest obstacles. According to the HIV Vaccines and Microbicides Resource Tracking Working Group, the total global investment in microbicide research and development was approximately US $\$ 226.5$ million in 2007, with the public sector providing 90\% (\$203.0 million) of funds [103]. This is compared with funding of $\$ 961$ million for HIV-vaccine-related research and development in the same year [103]. This huge discrepancy in funding is partly due to the difficulties experienced in mobilizing pharmaceutical industry support for microbicide research and development. Although funding of microbicide research has significantly increased over the years, a successful product will require extensive and sustained investment in research and development, access and advocacy [8]. In general, the product pipeline needs a very large number of products in Phase I owing to the high attrition rate before a product warrants assessment for efficacy against HIV infection. At present, the dearth of products in the preclinical and Phase I pipeline is a source of major concern.

There are also several logistical and ethical issues involved in the conduct of microbicide trials. Microbicides are typically tested in large numbers of sexually active people from selected populations that are likely to be at high risk of acquiring HIV infection. Thus, clinical trials are often carried out in developing countries that have high levels of infection [8]. This has raised concerns about the potential for exploitation of vulnerable populations; a concern that is, fortunately, not borne out of reality due to the high ethical and care standards maintained in all the current microbicides trials. Furthermore, microbicide trials are, in reality, being conducted in many countries throughout the world including Europe and the USA.

During the conduct of microbicide trials researchers are ethically obliged to promote and counsel on the use of proven HIV prevention options, such as condoms. Under these conditions the trial can only measure whether microbicides improve upon the protection afforded by existing proven prevention technologies, especially condom use [8]. Microbicides will also only work if they are widely accepted and used consistently by women. Measuring adherence is challenging and current clinical trials all include some form of assessment of product use, but these measures, such as self-report, can sometimes be unreliable. Other practical challenges that

Table 1. The current microbicide candidate pipeline.

\begin{tabular}{|c|c|c|c|}
\hline Phase & Candidate & Mechanism of action & Current status \\
\hline Phase III & $\begin{array}{l}\text { PRO 2000/5 gel } \\
\text { Tenofovir gel }\end{array}$ & $\begin{array}{l}\text { Entry/fusion inhibitor } \\
\text { Replication inhibitor }\end{array}$ & $\begin{array}{l}\text { Ongoing } \\
\text { Planned }\end{array}$ \\
\hline Phase IIB & $\begin{array}{l}\text { Tenofovir gel } \\
\text { Tenofovir gel }\end{array}$ & $\begin{array}{l}\text { Replication inhibitor } \\
\text { Replication inhibitor }\end{array}$ & $\begin{array}{l}\text { Ongoing } \\
\text { Planned }\end{array}$ \\
\hline Phase II/III & Invisible Condom ${ }^{\circledR}$ & Physical barrier and surfactant & Planned \\
\hline Phase I/II & VivaGel $^{\circledR \dagger}$ (SPL7013 gel) & Entry/fusion inhibitor & Ongoing \\
\hline Phase I & $\begin{array}{l}\text { Dapivirine }{ }^{\dagger} \text { (TMC120) gel } \\
\text { Ethanol in emollient gel } \\
\text { UC-781 } \\
\text { CAP vaginal soft tablet } \\
\text { MIV-150 gel }\end{array}$ & $\begin{array}{l}\text { Replication inhibitor } \\
\text { Surfactant } \\
\text { Replication inhibitor } \\
\text { Combination product } \\
\text { Combination product }\end{array}$ & $\begin{array}{l}\text { Ongoing } \\
\text { Ongoing } \\
\text { Ongoing } \\
\text { Planned } \\
\text { Planned }\end{array}$ \\
\hline
\end{tabular}


undermine the goals of a microbicide-effectiveness trial are behaviors, such as anal sex and use of other intravaginal substances.

A significant concern in the clinical assessment of microbicide effectiveness is the potential hazard related to reproductive toxicity. Women who become pregnant during the microbicide trial are required to discontinue product use, as the full effects of the product on a pregnancy are not well understood. Several microbicide studies have experienced high rates of pregnancies [104], which adversely affects the power and minimizes the effect size of the study outcome in an intention-to-treat analysis. This is because pregnant women who acquire HIV during the time when they are not using the product are counted as a microbicide 'failure'.

\section{Conclusion}

Although there have been a number of setbacks in the microbicide field, the results from the HPTN 035 study provide hope that the development of a safe and effective microbicide is possible. Despite the many challenges, microbicides provide a real potential to influence the course of the HIV epidemic and are likely to be available and accessible sooner than HIV vaccines and will fill an important gap for women-controlled prevention methods.

\section{Future perspective}

- If the MDP 301 study produces a similar level of PRO 2000 gel effectiveness as HPTN 035, data will be needed to address the main obstacles to widespread use, specifically, safety in pregnancy, long-term safety, safety in very high frequency use (beyond 28 applications in a fortnight), effectiveness against nonclade $\mathrm{C}$ HIV, approaches to increase over-the-counter access to the product, messaging to promote uptake and low-cost applicators with ease of disposal.

- Which formulation of microbicides will be most acceptable (gel, vaginal ring etc)?

- How should a microbicide product be formulated to promote adherence?

- How can the Phase I pipeline be expanded?

Financial \& competing interests disclosure

The authors are conducting the CAPRISA 004 trial of tenofovir gel, which is supported by the Centre for the AIDS Programme of Research in South Africa (CAPRISA), the USA Agency for International Development (USAID), Family Health International (FHI) (co-operative agreement \#GPO-A-00-0500022-00000, contract \#132119), CONRAD and LIFElab, a biotechnology centre of the South African Department of Science and Technology. Salim S Abdool Karim is protocol chair of the HPTN 035 study and member of the executive committee of the NIH-funded (grant \#AI068633) Microbicide Trials Network (MTN). He is also supported by NIH grants AI069469, AI51794 and TWO0231. The authors have no other relevant affiliations or financial involvement with any organization or entity with a financial interest in or financial conflict with the subject matter or materials discussed in the manuscript apart from those disclosed.

No writing assistance was utilized in the production of this manuscript. “...the total global

investment in microbicide research and

development was approximately

US\$226.5 million in 2007

with the public sector providing $90 \%$ (\$203 million) of funds."

\section{Bibliography}

1. Kreiss J, Ngugi E, Holmes K et al.: Efficacy of nonoxynol 9 contraceptive sponge use in preventing heterosexual acquisition of HIV in Nairobi prostitutes. JAMA 268, 477-482 (1992).

2. Roddy RE, Zekeng L, Ryan KA, Tamoufe U, Weir SS, Wong EL: A controlled trial of nonoxynol 9 film to reduce male-to-female transmission of sexually transmitted diseases. N. Engl. J. Med. 339, 504-510 (1998).

3. van Damme L, Ramjee G, Alary M et al.: Effectiveness of COL-1492, a nonoxynol-9 vaginal gel, on HIV-1 transmission in female sex workers: a randomised controlled trial. Lancet 360, 971-977 (2002).

4. Feldblum PJ, Adeiga A, Bakare R et al.: SAVVY vaginal gel (C31G) for prevention of HIV infection: a randomized controlled trial in Nigeria. PLoS ONE 3, e1474 (2008).
5. Horwood J: Cellulose sulphate microbicide trial halted. Lancet Infect. Dis. 7, 183-183 (2007).

6. Skoler-Karpoff S, Ramjee G, Ahmed K et al: : Efficacy of Carraguard for prevention of HIV infection in women in South Africa: a randomised, double-blind, placebo-controlled trial. Lancet 372, 1977-1987.

7. Abdool Karim SS, Coletti A, Richardson B et al:: Safety and effectiveness of vaginal microbicides buffergel and 0.5\% PRO 2000/5 gel for the prevention of HIV infection in women: results of the HPTN 035 trial [abstract 48LB]: Presented at: 16th Conference on Retroviruses and Opportunistic Infections. Montreal, Canada, 8-11 February 2009.

8. Abdool Karim SS, Baxter C: New prevention strategies under development/ investigation. In: HIV/AIDS in South Africa. Abdool Karim SS,
Abdool Karim Q (Eds). Cambridge University Press, Cape Town, South Africa, 226-240 (2005).

9. $\quad$ Viread $^{\circledR}$, package insert. Gilead Sciences, Inc., CA, USA.

\section{Websites}

101. Alliance for microbicide development, April 2009 pipeline update. www.microbicide.org/cs/pipeline (Accessed 8 April 2009).

102. HPTN 035 protocol team, protocol: HPTN 035 Phase II/IIb safety and effectiveness study of the vaginal microbicides buffergel and 0.5\% PRO 2000/5 gel (P) for the prevention of HIV infection in women. Version 3.0.

www.hptn.org/web\%20documents/HPTN_ Protocols/HPTN035/HPTN035v3.pdf (Accessed 12 December 2006). 


\section{Editorial Abdool Karim \& Baxter}

103. The HIV vaccines and microbicides resource tracking working group, sustaining the HIV prevention research agenda: funding for research and development of HIV vaccines, microbicides and other new prevention options (2000-2007).

www.hivresourcetracking.org

(Accessed 8 April 2009).
104. Smart T: Report on microbicides 2006: high rates of pregnancy pose challenges for microbicide trials. www.aidsmap.com/en/news/3F76DCE383FA-4523-A920-5CB0829DD33A.asp (Accessed 8 April 2009).

105. Alliance for microbicide development tables on ongoing and planned clinical trials. www.microbicide.org (Accessed 8 April 2009).

\section{Affiliations}

- Salim S Abdool Karim

Department of Epidemiology,

Columbia University, NY, USA and

CAPRISA, University of KwaZulu-Natal, Durban, South Africa,

Tel.: +27312604550

Fax: +27312604549

karims1@ukzn.ac.za

- Cheryl Baxter

CAPRISA, University of KwaZulu-Natal, Durban, South Africa,

baxterc1@ukzn.ac.za 